\title{
Ubiquitin and Drug Discovery: Challenges and Opportunities
}

\author{
Allan M. Weissman
}

Published online: 11 June 2013

(C) Springer Science+Business Media New York (outside the USA) 2013

ATP-dependent protein degradation in reticulocytes was demonstrated by Alfred Goldberg in 1977. This was followed, almost immediately, by the discovery of the ubiquitin system for proteolysis by Avram Hershko, Aaron Ciechanover and Irwin Rose. Characterization of the proteasome began soon thereafter. Prior to these seminal discoveries the prevailing concept was that whatever protein degradation was occurring in cells was lysosomal.

Naturally-occurring cellular substrates for the ubiquitinproteasome system (UPS) began to be characterized in 1987. Beginning in the mid 1990s, there was an explosion in identified UPS targets, in part due to development of proteasome inhibitors. It is now universally appreciated that regulated protein degradation through the UPS is a rapid and exquisitely regulated means of controlling the levels of myriad cellular proteins and disposing of abnormal ones. In addition to proteasomal targeting, ubiquitin plays even broader roles in proteostasis, as it is integral to lysosomal targeting and autophagy (see review this issue by Cuervo and colleagues).

The roles of ubiquitin in cells extend far beyond protein degradation. Protein ubiquitination is critical to processes such as DNA repair, NF- $\kappa \mathrm{B}$ activation and transcription. Understanding how a single molecule conjugated to different positions on proteins and in different linkages can transmit such disparate signals remains a major challenge. We also now appreciate that ubiquitin is not the sole covalent polypeptide modifier of proteins. Ubiquitin-like modifiers are targeted to proteins through similar multi-enzyme

A. M. Weissman ( $\bowtie)$

Laboratory of Protein Dynamics and Signaling,

Center for Cancer Research, National Cancer Institute,

Frederick, MD 21702-1201, USA

e-mail: amw@nih.gov cascades and many of these functionally intersect with ubiquitin.

Given the broad spectrum of UPS enzymes and substrates, it is not surprising that the UPS is implicated in diseases ranging from cancer to neurodegenerative disorders to infectious diseases, such as HIV. Its importance in cancer is borne out through the use of the proteasome inhibitor bortezomib for treatment of multiple myeloma and other lymphoid malignancies, and the recent FDA approval of carfilzomib for relapsed and refractory multiple myeloma.

There is, of course, intense interest in targeting enzymes involved in ubiquitination. Studies from our lab established proof of principle for inhibiting the ubiquitin E1 in vitro and in cells. Moving beyond this to therapeutics is, however, a major challenge. One area of promise is in the development of agents that inhibit the Nedd8 E1. These could potentially alter the fate and function of the numerous cullin RING ligase substrates, many of which are implicated in the cell cycle and other critical processes (see article this issue by Liao and colleagues). Such an agent, MLN4924, is now in Phase I clinical trials for both solid tumors and hematologic malignancies. By employing the principles and lessons learned in targeting the Nedd8 E1, there is the very real potential to develop potent inhibitors specific for the E1s for ubiquitin and other ubiquitin-like modifiers. However, as is the case with proteasome inhibitors, the large number of proteins affected by such inhibitors makes it difficult a priori to predict therapeutic outcomes.

Substrate specificity in the ubiquitin system lies largely with the over 600 ubiquitin ligases (E3s), which act in combination with subsets of the $\sim 40$ ubiquitin-conjugating enzymes (E2s). Their activity is opposed by $\sim 100$ deubiquitinating enzymes (DUBs). A challenge in drug 
development therefore lies not only in determining how to target these proteins, but also in how to do so with adequate specificity. RING-type E3s, HECT E3s, and E2s each have marked structural similarity within each family. Thus, as one moves beyond strategies aimed at disrupting specific E3-substrate interactions, as exemplified by the Nutlins that disrupt interactions between the tumor suppressor p53 and its $\mathrm{E} 3, \mathrm{Mdm} 2 / \mathrm{Hdm} 2$, approaches to targeting the intrinsic ligase activity of specific E3s or their cognate E2s become problematic. However, the potential for such targeting in the case of $\mathrm{Mdm} 2 / \mathrm{Hdm} 2$ was provided early on by several groups, including our collaborators and us. As combinatorial pairs of E2s and E3s are matched with particular substrates and structural nuances important for function become apparent, we will hopefully be on the way to developing potent drugs with a high level of specificity for individual E2s, E3s, or E2-E3 pairs.

Issues of specificity, as well as targetability, also pertain to the multiple classes of DUBs. There is already immense interest in identifying both structural and functional differences among DUBs and in targeting these enzymes. This is evident both from these proceedings and from presentations at the 4th Ubiquitin Drug Discovery and Diagnostic Conference in 2012.

Beyond the proteasome and enzymes of the ubiquitin system, ubiquitin receptors and chaperones represent other classes of promising targets. Most notable among these is the hexameric AAA-ATPase p97/VCP (Cdc48 in yeast), for which inhibitors have now been developed.

Overall, therapeutics developed from the target-rich ubiquitin system have the potential to greatly enhance personalized medicine and treat previously intractable human diseases.

The reviews and primary research featured in this issue are representative of the exciting presentations at the 4th Ubiquitin Drug Discovery and Diagnostic Conference. ${ }^{1}$ Aside from their intrinsic scientific interest, they collectively underscore the great interest in, and energy being devoted to, both advancing our basic knowledge and moving toward therapeutic development.

\footnotetext{
1 VLI-Research is the founder and organizer of annual Ubiquitin Drug Discovery and Diagnostic Conferences. VLI-Research is devoted to organizing conferences to advance both scientific knowledge and therapeutic development related to the ubiquitin pathway.
} 